\title{
Effectiveness of pertussis vaccines for adolescents and adults: case-control study
}

\author{
(c) $(1)(9)$ OPEN ACCESS
}

\author{
Roger Baxter codirector, Joan Bartlett analyst/programmer, Ali Rowhani-Rahbar vaccine safety \\ fellow, Bruce Fireman statistician, Nicola P Klein codirector
}

Kaiser Permanente Vaccine Study Center, 1 Kaiser Plaza, Oakland, CA 94612, USA

\begin{abstract}
Objective To assess the effectiveness of reduced acellular pertussis (Tdap) vaccines in adolescents and adults.

Setting Kaiser Permanente Northern California.

Design Case-control study.

Participants All polymerase chain reaction (PCR) confirmed cases of pertussis in members aged 11 years and older from January 2006 to December 2011. We compared the Tdap vaccination status of PCR positive cases with two control groups: people testing negative for pertussis by PCR and closely matched people from the general Kaiser Permanente Northern California population.
\end{abstract}

Main outcome measure PCR confirmed pertussis. The association of Tdap vaccination with the odds of pertussis infection was estimated by conditional logistic regression, with adjustment for calendar time, pertussis vaccine type received in early childhood, age, sex, race or ethnic group, and medical clinic. We calculated Tdap vaccine effectiveness as 1 minus the adjusted odds ratio.

Results The study population included 668 PCR positive cases, 10098 PCR negative controls, and 21599 Kaiser Permanente Northern California matched controls. Tdap vaccination rates were $24.0 \%$ in PCR positive cases and $31.9 \%$ in PCR negative controls $(P<0.001)$. The adjusted estimate of effectiveness of Tdap vaccination against pertussis was $53.0 \%$ (95\% confidence interval $41.9 \%$ to $62.0 \%$ ) in the comparison with PCR controls, and $64.0 \%$ (55.5\% to $70.9 \%$ ) in the comparison with Kaiser Permanente Northern California controls.

Conclusion Tdap vaccination was moderately effective at preventing PCR confirmed pertussis among adolescents and adults.

\section{Introduction}

Pertussis is a worldwide, cyclic illness, which was concentrated in children under 5 years of age during the pre-vaccine era. After widespread use of whole cell pertussis, tetanus, and diphtheria toxoid vaccines for infants starting in the United States in 1948, the incidence of pertussis decreased noticeably and infants under 6 months and adolescents became the most susceptible age groups. ${ }^{12}$ Though infants experience most of the mortality from pertussis, adolescents and adults serve as reservoirs and vectors of infection ${ }^{2-5}$ and comprise about half of all cases. ${ }^{6}$ During the 1990s, safety concerns regarding whole cell pertussis vaccines prompted the United States to switch to acellular pertussis vaccines. Despite high vaccine coverage in infants and children, since the 1980s the United States has experienced periodic outbreaks of pertussis, with incidence increasing over time. ${ }^{17-9}$ Reasons for the increase are likely varied, but studies of recent outbreaks have found that acellular pertussis vaccines for children are less effective than earlier whole cell formulations, ${ }^{70-12}$ and that protection wanes substantially after the last dose. ${ }^{13} 14$

Tetanus toxoid, reduced diphtheria toxoid, and reduced acellular pertussis (Tdap) vaccines were developed to improve protection against pertussis among adolescents and adults. Two Tdap vaccines were licensed in 2005 on the basis of immunogenicity measures comparable to the acellular pertussis vaccines already approved for infants, ${ }^{15}{ }^{16}$ despite uncertainty about the relation between these measures and effectiveness. ${ }^{17}{ }^{18}$ That same year, the US Advisory Committee on Immunization Practices recommended a single dose of Tdap to replace the next scheduled tetanus diphtheria (Td) booster for all people aged $11-64,{ }^{19}$ and in 2012 the committee extended its recommendation for routine Tdap vaccination to all people aged 65 and older. ${ }^{20}$

Post-licensure studies of the Tdap vaccines showed high effectiveness, ${ }^{21-23}$ but the studies were limited by small numbers of pertussis cases and by use of populations who had all received whole cell pertussis vaccines as children. Studies to date provide little or no information as to the effectiveness of the Tdap booster when administered to the newly emerging cohort of adolescents previously vaccinated entirely with acellular pertussis instead of whole cell pertussis vaccines.

In 2010 the incidence of pertussis in California rose to its highest level in the past 50 years. ${ }^{24}{ }^{25}$ With data from a six year period including this outbreak, we assessed the effectiveness of Tdap vaccination in reducing the risk of pertussis among adolescents and adults who had received whole cell pertussis vaccines as children as well as among younger adolescents who had only ever received acellular pertussis vaccines. 


\section{Methods}

\section{Study population and setting}

Kaiser Permanente of Northern California is an integrated healthcare delivery system serving 3.2 million members across 16 counties in California. The membership reflects the racial and economic diversity of the general population in Northern California, though, as a commercially insured population, it under-represents people with very low incomes. ${ }^{26-28}$ Members obtain almost all their medical care at Kaiser Permanente Northern California facilities, including 49 outpatient medical clinics and 19 hospitals. Kaiser Permanente Northern California databases store detailed information on all encounters, diagnoses, medications, vaccinations, and laboratory tests, as well as information on enrollment and demographics, which are linked using the patient's unique medical record number.

The medical record includes data on race or ethnic group for approximately $80 \%$ of members. For the rest, we imputed race or ethnic group using the RAND BISG algorithm, ${ }^{29}$ which uses information on surname and home address to infer the probability of being in each of six race or ethnic groups.

All microbiology tests are conducted in Kaiser Permanente Northern California's central laboratory. Starting in late 2005, the central laboratory replaced culture and fluorescent antibody methods with real time polymerase chain reaction (PCR) for detection of Bordetella pertussis and B parapertussis. Nasopharyngeal swabs for testing are collected by physicians and nurses in clinical areas that are distant from the injection clinics where children and adults receive their vaccines. Pertussis PCR test results are reported as positive for $B$ pertussis, positive for $B$ parapertussis, or negative for both $B$ pertussis and $B$ parapertussis.

\section{Study design}

We conducted a case-control study to examine the association of Tdap vaccination and pertussis infection among people 11 years and older. We defined cases as people who tested PCR positive for pertussis during the study period from January 2006 to December 2011. We compared the Tdap immunization status of these cases with that of two control groups. The first control group consisted of people who tested PCR negative for pertussis during the study period. The second control group was selected from the general Kaiser Permanente Northern California membership. For each case we selected a set of matched controls comprised of all Kaiser Permanente Northern California members who were enrolled on the date the case tested PCR positive and for whom sex, age (year and quarter of birth), race or ethnic group, and medical clinic were the same, and who were inferred - on the basis of birth year - to have received the same pertussis vaccine type (whole cell or acellular) as infants. We determined whether each participant had received Tdap at any time prior to their PCR test date. We established the Tdap status of Kaiser Permanente Northern California controls on the PCR test date of their matched case. We did not distinguish between the two brands of Tdap vaccine used during the study period: Boostrix (GlaxoSmithKline, Rixensart, Belgium) and Adacel (Sanofi Pasteur, Swiftwater, PA).

For both cases and controls we excluded those less than 11 years of age on the PCR test date because Tdap coverage was low ( $2 \%$ in 10 year olds and negligible below age 10 ). We excluded those who had the PCR test within one week after their Tdap vaccine, to allow time for the booster immune response. We also excluded those with more than a three month gap in membership between October 2005 (when Tdap became available in Kaiser Permanente Northern California) or their 11 th birthday, whichever was later, and their PCR test date to ensure complete vaccination history and correct classification of Tdap status. We excluded any case as a subsequent control.

\section{Statistical analyses}

We used conditional logistic regression to estimate the association of Tdap vaccination with the odds of pertussis infection. We estimated the odds ratio- the odds of pertussis in vaccinated people divided by the odds of pertussis in unvaccinated people - in separate comparisons of the cases with each of the control groups.

Comparing the cases with PCR negative controls, we first estimated the odds ratio without adjustment for covariates. We then calculated the adjusted odds ratio by conditioning on calendar time and the type of pertussis vaccines received in early childhood, and including covariates to adjust for age, sex, race or ethnic group, and medical clinic (49 clinics aggregated into 12 service areas). The width of the calendar time intervals varied from yearly when cases were few to monthly during the epidemic period.

Comparing the cases with Kaiser Permanente Northern California matched controls, much finer stratification was possible, using the matching variables: PCR test date, childhood pertussis vaccines, age, sex, race or ethnic group, and medical clinic. The only covariates in addition to Tdap status were the set of six imputed probabilities of race or ethnic group, to provide additional adjustment for the people whose race or ethnicity was missing from the Kaiser Permanente Northern California database.

We defined five categories of inferred exposure to whole cell or acellular pertussis vaccines in childhood, based largely on year of birth. The birth year parameters were derived from Kaiser Permanente Northern California data on the timing of the transition from whole cell to acellular pertussis vaccines, which began in 1991 for the fifth dose and was completed for all five childhood doses by 1999 . The last transition from whole cell to acellular pertussis vaccines occurred during the birth cohorts 1996-98 for the three infant doses. We grouped all individuals as follows: pre-vaccine era before widespread pertussis vaccination (born before 1950); all whole cell pertussis vaccines (born 1950-85); first three doses whole cell pertussis vaccines (born 1986-95, or born 1996-98 with confirmed whole cell history); first three doses unknown or mix of whole cell and acellular pertussis vaccines (born 1996-98 and data not available, or confirmed mix); or all acellular pertussis vaccines (born after 1998, or born 1996-98 with confirmed acellular history). We obtained data on vaccine type from electronic databases for those born 1996-98 who had received their first three doses at Kaiser Permanente Northern California.

We calculated the effectiveness of Tdap vaccine as 1 minus the adjusted odds ratio estimated from the conditional logistic regression model. Separate estimates of vaccine effectiveness were calculated based on the results for PCR negative and Kaiser Permanente Northern California controls.

We also estimated Tdap vaccine effectiveness in three subgroups of people with different pertussis vaccination histories (those born before pertussis vaccine was widely available, those who had received all whole cell pertussis vaccines in early childhood, and those who had received all acellular pertussis vaccines in early childhood), using the same approach described for the entire study population.

All statistical analyses were performed using SAS software, version 9.2. 


\section{Results}

During the study period from January 2006 to December 2011, a total of 30004 pertussis PCR tests were performed on Kaiser Permanente Northern California members of all ages, and 1598 (5.3\%) were positive for pertussis. Figure $1 \Downarrow$ depicts the results of PCR testing and how PCR positive cases and PCR negative controls were selected for the study. Rates of Tdap vaccination among those 11 years and older steadily increased over these six years, rising from near $0 \%$ in early 2006 to between $25 \%$ and $30 \%$ in early 2010 when the pertussis outbreak started (fig $2 \Downarrow)$.

During the pertussis outbreak from January 2010 through June 2011 (which includes nearly $75 \%$ of all cases in the study population), the incidence of pertussis in the entire Kaiser Permanente Northern California population was strongly related to age. Incidence increased sharply after age 5 , was highest at ages 10 and 11 , then decreased sharply until age 15 , and was low in adults. Decreasing pertussis rates from ages 11 and up coincided both with the use of Tdap vaccines and with receipt of whole cell pertussis vaccines during infancy (fig $3 \Downarrow$ ). At the epidemic's peak, Tdap vaccination rates were near $0 \%$ until age 10 , then rose rapidly reaching a high of $72 \%$ at age 15 , then decreased to between $25 \%$ and $35 \%$ for ages $21-64$, and to $10 \%$ for ages 65 and older (fig 3).

The case-control analysis compared $668 \mathrm{PCR}$ positive pertussis cases with 10098 PCR negative controls and with 21599 Kaiser Permanente Northern California controls. The range in the number of matched controls to each case was 1 to 230 . Of the 668 cases in the overall study population, $17(2.5 \%)$ had one matched control, $41(6.1 \%)$ had 2-4 controls, $76(11.4 \%)$ had 5-9 controls, and $534(79.9 \%)$ had 10 or more controls. Table $1 \Downarrow$ describes the characteristics of those who underwent PCR testing for pertussis. Cases and PCR negative controls differed significantly with respect to PCR test date, pertussis vaccination history, age, sex, race or ethnic group, and medical clinic. For Kaiser Permanente Northern California controls, matching ensured that cases and controls were identical for all of these characteristics except age, where they were very similar.

Tdap vaccination coverage was $24.0 \%$ for PCR positive cases versus $31.9 \%$ for $\mathrm{PCR}$ negative controls $(\mathrm{P}<0.001)$. The percentage of recipients of Tdap vaccines who had been vaccinated within the previous two years was similar for PCR positive cases (55.6\%) and PCR negative controls (57.2\%). Comparing cases with PCR negative controls, the unadjusted odds ratio was 0.68 (95\% confidence interval 0.56 to 0.81 ). After controlling for calendar time, pertussis vaccine type received in early childhood, age, sex, race or ethnic group, and medical clinic, the adjusted odds ratio was 0.47 (0.38 to 0.58 ). Comparing cases with Kaiser Permanente Northern California matched controls, the adjusted odds ratio was 0.36 (0.29 to $0.45)$.

The estimated effectiveness of Tdap vaccination against pertussis, derived from the adjusted odds ratio, was 53.0\% ( $41.9 \%$ to $62.0 \%, \mathrm{P}<0.001$ ) when comparing cases with PCR negative controls and $64.0 \%(55.5 \%$ to $70.9 \%, \mathrm{P}<0.001)$ when comparing cases with Kaiser Permanente Northern California controls.

Within the subgroups defined by childhood pertussis vaccination history, the estimates for effectiveness of the Tdap vaccine were broadly similar to those for the full study population in the whole cell and acellular pertussis vaccines subgroups, but substantially lower in the pre-vaccine subgroup. The reduction in pertussis risk conferred by Tdap vaccination was statistically significant for the whole cell and acellular pertussis vaccines subgroups but not for the pre-vaccine subgroup in comparisons with both control groups (table $2 \Downarrow$ ). The subgroups differed in age, Tdap vaccination coverage, and recency of Tdap vaccination. The acellular subgroup was younger (mean age in PCR tested, 11.4 years) than the whole cell (43.3 years) and pre-vaccine (68.9 years) subgroups. Tdap vaccination coverage was higher in the acellular (32.0\%) and whole cell (27.7\%) subgroups than in the pre-vaccine (17.4\%) subgroup. Among recipients of Tdap vaccines, the percentage vaccinated within two years of their PCR test was greater in the acellular $(91.6 \%)$ subgroup than in the whole cell or pre-vaccine ( $61.7 \%$ and $57.9 \%$, respectively) subgroups.

\section{Clinical severity}

Most of the pertussis cases seemed to be mild or moderate in severity. Within five days before or after the PCR test, 647 out of 668 cases (96.9\%) had an outpatient visit and 613 (91.8\%) received a macrolide prescription (almost all azithromycin); 497 cases $(74.4 \%)$ received a diagnosis of exposure to whooping cough, cough, or pertussis, and another $143(21.4 \%)$ received a related diagnosis (that is, respiratory tract infection, bronchitis, asthma, pneumonia, and unspecified viral infections). Review of all admissions to hospital and emergency room visits within 100 days before or after the PCR test revealed that eight cases (1.2\%) were admitted for pertussis, all within one week of the test, and 60 cases $(9.0 \%)$ had 79 emergency room visits related to pertussis, with all but one visit occurring within one month of the test.

\section{Discussion}

We found that tetanus toxoid, reduced diphtheria toxoid, and reduced acellular pertussis (Tdap) vaccination was moderately effective at preventing pertussis in people aged 11 years and older during a study period that included the large outbreak in California in 2010 and 2011. Tdap vaccination was estimated to reduce the risk of polymerase chain reaction (PCR) confirmed pertussis in our study population by $53 \%$ (95\% confidence interval $42 \%$ to $62 \%$ ) in the comparison with PCR negative controls and by $64 \%$ (56\% to $71 \%$ ) in the comparison with Kaiser Permanente Northern California controls. Both estimates of vaccine effectiveness and the range covered by their confidence intervals indicate that Tdap vaccination was moderately protective against pertussis.

In subgroup analyses, we found that the Tdap booster was moderately effective both in older people who had received all whole cell pertussis vaccines as infants and in younger people who had received all acellular pertussis vaccines. Yet, rates of pertussis were highest in the acellular subgroup consisting of young adolescents ages 11-14, probably because protection from their childhood doses waned substantially over time. ${ }^{13} 30$ As a result, the absolute benefit of Tdap vaccination was much greater for people who had received acellular instead of whole cell vaccines as children. Strategies to decrease the incidence of pertussis should prioritize giving the Tdap booster to people who received only acellular pertussis vaccines as children, as California has done since the 2010 outbreak, requiring Tdap vaccination for middle school students. To our knowledge this is the first study to assess the effectiveness of the Tdap booster in members of this new generation that has received all acellular vaccines.

We had limited power to assess the effectiveness of Tdap vaccines in the subgroup of people born before 1950. The confidence interval suggests that effectiveness was unlikely to be higher than $68 \%$. This subgroup was at low risk of pertussis, 
perhaps as a result of immunity conferred by natural infection, receipt of single antigen whole cell vaccines available before the whole cell pertussis combination vaccines, or less frequent contact with infected people; this subgroup also had low Tdap vaccination coverage, since it was not until 2012 that the US Advisory Committee on Immunization Practices recommended routine Tdap vaccination for people aged 65 years and older.

Prior post-licensure studies of Tdap vaccine effectiveness have been limited by low numbers of cases. One controlled study showed vaccine effectiveness of $92 \%$ ( $95 \%$ confidence interval $32 \%$ to $99 \%$ ) in preventing clinical pertussis, ${ }^{21}$ but there were only 10 people who met the definition of a primary case. More recently, another study investigating a pertussis outbreak in the US Virgin Islands found vaccine effectiveness of $66 \%^{22}$ in an analysis of 51 confirmed or probable pertussis cases. An observational study from Australia ${ }^{23}$ evaluating a mass vaccination program for high school students, found vaccine effectiveness of $78.0 \%$ (95\% confidence interval $60.7 \%$ to $87.6 \%$ ) based on 167 cases.

\section{Strengths and limitations of this study}

One strength of our study was the large number $(n=668)$ of PCR confirmed cases of pertussis. Another strength was that we estimated the effectiveness of Tdap vaccines separately using two sets of controls. Comparisons with both control groups support our finding of moderate effectiveness. Our use of two control groups adds to our confidence in ruling out very high effectiveness, but also adds uncertainty about the precise level of effectiveness. Each control group had different strengths and weaknesses in terms of mitigating bias. On the one hand, PCR negative controls were probably more similar to the PCR positive cases than Kaiser Permanente Northern California controls in their propensity to seek care. Care seeking may be an important source of confounding since it is related to the likelihood of receiving both a PCR test and Tdap vaccination. On the other hand, Kaiser Permanente Northern California's large population allowed us to precisely match controls to cases, and Kaiser Permanente Northern California controls were more similar to cases than were PCR negative controls on all measured covariates. Some of these covariates were important confounders as evidenced by the large increase in the adjusted compared with the unadjusted estimates for vaccine effectiveness $(53 \% \mathrm{v}$ $32 \%$ ) in the analysis with PCR negative controls. Studies of acellular pertussis vaccines for infants have shown that estimated effectiveness can vary with the criteria used to identify pertussis cases. In general, the vaccines seem to be more effective as the case definition becomes stricter (that is, more specific) and requires higher severity. ${ }^{31}$ Since we included mild cases of pertussis in our study, protection against more severe disease may be greater than indicated by our results. However, for this particular vaccine, intended more to prevent spread of pertussis to infants than to avert illness in vaccinees themselves, prevention of even mild disease is important.

Our results reflect the average effectiveness of Tdap vaccination over all available follow-up time. We were not able to assess whether effectiveness waned over time because the vaccine is relatively new. In the subgroup who received all acellular pertussis vaccines as infants, $92 \%$ of Tdap recipients were vaccinated within two years prior to PCR testing. Recent studies have found early waning of immunity for the childhood acellular pertussis vaccines, ${ }^{13} 3032$ and future studies should examine whether there could be waning for the Tdap booster as well.

Our study has limitations. As an observational study, it is subject to confounding from unmeasured factors related to both Tdap vaccination and pertussis infection, such as the presence of pertussis infection in the household. The potential for confounding may have been greater among people aged 65 and older in whom Tdap vaccine was not routinely administered; in this group, vaccination may have been related to perceived risk, which could lead to an underestimate of vaccine effectiveness. If vaccinated cases were milder and care was delayed, this could result in more cases of pertussis who are PCR negative, leading to an underestimate of vaccine failures, and a corresponding overestimate of vaccine effectiveness, especially in the comparison of cases with PCR negative controls. In addition, problems with sensitivity or specificity of the PCR test that are non-differential (that is, not related to vaccination status) would lead to an underestimate of vaccine effectiveness. $^{33}$

\section{Conclusion}

In conclusion, Tdap vaccination was moderately protective against pertussis in the setting of a large outbreak in California. Tdap was moderately effective for those vaccinated with either whole cell or acellular pertussis agents as infants, though the acellular subgroup was more in need of protection. Our findings draw attention to the need for more effective vaccines to prevent pertussis outbreaks.

Contributors: RB had full access to all of the data in the study and takes responsibility for the integrity of the data and the accuracy of the data analysis. RB supervised the project, assisted in design and analysis, and crafted the draft manuscript. JB extracted all data, performed analyses, assisted in design, and critically reviewed the manuscript. AR assisted in design, interpretation of analyses, and critically reviewed the manuscript. BF led the statistical work, assisted in design and analyses, and critically reviewed the manuscript. NK assisted in design, interpretation of analyses, and critically reviewed the manuscript.

Funding: This study was funded by Kaiser Permanente. There was no support from any other organizations for this work.

Competing interests: All authors have completed the ICMJE uniform disclosure form at www.icmje.org/coi_disclosure.pdf and declare: RB and NPK have received research grants from Sanofi Pasteur, GlaxoSmithKline, Merck, Novartis, Pfizer, and Medlmmune for unrelated studies; no other relationships or activities that could appear to have influenced the submitted work.

Ethical approval: This study was approved by the Kaiser Permanente Northern California institutional review board.

Data sharing: No additional data available.

Plotkin SA, Orenstein WA, Offit PA. Vaccines. 5th ed. Saunders Elsevier, 2008.

2 Cherry JD. Epidemiology of pertussis. Pediatr Infect Dis J 2006;25:361-2.

3 Wendelboe AM, Njamkepo E, Bourillon A, Floret DD, Gaudelus J, Gerber M, et al. Transmission of Bordetella pertussis to young infants. Pediatr Infect Dis J 2007;26:293-9.

4 Bisgard KM, Pascual FB, Ehresmann KR, Miller CA, Cianfrini C, Jennings CE, et al. Infant pertussis: who was the source? Pediatr Infect Dis J 2004;23:985-9.

5 Lavine J Broutin $\mathrm{H}$, Harvill ET, Bjornstad ON. Imperfect vaccine-induced immunity and whooping cough transmission to infants. Vaccine 2011;29:11-6.

6 Pertussis outbreak among adults at an oil refinery-Illinois, August-October 2002. MMWR Morb Mortal Wkly Rep 2003;52:1-4.

7 Cherry JD. The epidemiology of pertussis: a comparison of the epidemiology of the disease pertussis with the epidemiology of Bordetella pertussis infection. Pediatrics 2005;115(5):1422-7.

8 Pertussis epidemic - washington, 2012. MMWR Morb Mortal Wkly Rep 2012;61:517-22.

9 Nennig ME, Shinefield HR, Edwards KM, Black SB, Fireman BH. Prevalence and incidence of adult pertussis in an urban population. JAMA 1996;275:1672-4.

10 Sheridan SL, Ware RS, Grimwood K, Lambert SB. Unexpectedly limited durability of immunity following acellular pertussis vaccination in preadolescents in a North American outbreak. Clin Infect Dis 2012;55:1434-5.

11 Olin P, Rasmussen F, Gustafsson L, Hallander HO, Heijbel H. Randomised controlled trial of two-component, three-component, and five-component acellular pertussis vaccines compared with whole-cell pertussis vaccine. Ad Hoc Group for the Study of Pertussis Vaccines. Lancet 1997;350:1569-77.

12 Klein N, Bartlett J, Fireman B, Rowhani-Rahbar A, Baxter R. Comparative effectiveness of acellular versus whole cell pertussis vaccines in teenagers. Pediatrics 2013; published online 20 May; doi: 10.1542/peds.2012-3836. 


\section{What is already known on this topic}

Acellular pertussis vaccines for adolescents and adults are recommended in the United States

Worsening outbreaks of pertussis have led to questions regarding the effectiveness of acellular vaccines

Recent studies have shown that the protection from acellular pertussis vaccines given in childhood wanes substantially over time

\section{What this study adds}

Reduced acellular pertussis (Tdap) vaccines are moderately effective, reducing the risk of pertussis by about $60 \%$, and this effect is not diminished in those who received only acellular rather than whole cell vaccines, as infants.

Though these vaccines provide protection, more effective vaccines may be necessary to prevent further outbreaks

13 Klein NP, Bartlett J, Rowhani-Rahbar A, Fireman B, Baxter R. Waning protection after fifth dose of acellular pertussis vaccine in children. N Engl J Med 2012:367:1012-9.

14 Misegades LK, Winter K, Harriman K, Talarico J, Messonnier NE, Clark TA, et al. Association of childhood pertussis with receipt of 5 doses of pertussis vaccine by time since last vaccine dose, California, 2010. JAMA 2012;308:2126-32.

15 Administration. FFaD. Proper name: tetanus toxoid, reduced diphtheria toxoid and acellular pertussis vaccine, adsorbed. Tradename: Adacel. Manufacturer: Sanofi Pasteur, License No 1726. In: Department of Health and Human Services CfBEaR, ed. Package insert, 2005 .

16 Administration. FFaD. Tetanus toxoid, reduced diphtheria toxoid and acellular pertussis vaccine, adsorbed. Tradename: Boostrix. Manufacturer: GlaxoSmithKline Biologicals, License No 1617. In: US Department of Health and Human Services CfBEaR, ed. Package insert, 2005.

17 Plotkin SA. Vaccines: correlates of vaccine-induced immunity. Clin Infect Dis 2008:47:401-9.

18 Cherry JD, Gornbein J, Heininger U, Stehr K. A search for serologic correlates of immunity to Bordetella pertussis cough illnesses. Vaccine 1998;16:1901-6.

19 Kretsinger K, Broder KR, Cortese MM, Joyce MP, Ortega-Sanchez I, Lee GM, et al. Preventing tetanus, diphtheria, and pertussis among adults: use of tetanus toxoid, reduced diphtheria toxoid and acellular pertussis vaccine recommendations of the Advisory Committee on Immunization Practices (ACIP) and recommendation of ACIP, supported by the Healthcare Infection Control Practices Advisory Committee (HICPAC), for use of Tdap among health-care personnel. MMWR Recomm Rep 2006;55(RR-17):1-37.

20 Updated recommendations for use of tetanus toxoid, reduced diphtheria toxoid, and acellular pertussis (Tdap) vaccine in adults aged 65 years and older-Advisory Committee on Immunization Practices (ACIP), 2012. MMWR Morb Mortal Wkly Rep 2012;61:468-70.

21 Ward J, Cherry JD, Chang S, Partridge S, Lee H, Treanor J, et al. Efficacy of an acellular pertussis vaccine among adolescents and adults. N Engl J Med 2005;353:1555-63.

22 Wei SC, Tatti K, Cushing K, Rosen J, Brown K, Cassiday P, et al. Effectiveness of adolescent and adult tetanus, reduced-dose diphtheria, and acellular pertussis vaccine against pertussis. Clin Infect Dis 2010;51:315-21.

23 Rank C, Quinn HE, McIntyre PB. Pertussis vaccine effectiveness after mass immunization of high school students in Australia. Pediatr Infect Dis J 2009;28:152-3.

24 Reidmann E. Pertussis outbreak in California. Hum Vaccin 2010;6:684
25 Roehr B. Whooping cough outbreak hits several US states. BMJ 2010;341:c4627.

26 Gordon NP. Similarity of the adult Kaiser Permanente membership in Northern California to the insured and general population in Northern California: Statistics from the 2007 California Health Interview Survey. Internal Division of Research report. Kaiser Permanente Division of Research. 2012.

27 Krieger N. Overcoming the absence of socioeconomic data in medical records: validation and application of a census-based methodology. Am J Public Health 1992;82:703-10.

28 Selby JV Smith DH, Johnson ES, Raebel MA, Friedman GD, McFarland BH., ed. Kaiser Permanente Medical Care Program. 4th ed. Wiley, 2005.

29 Elliott MN, Fremont A, Morrison PA, Pantoja P, Lurie N. A new method for estimating race/ethnicity and associated disparities where administrative records lack self-reported race/ethnicity. Health Serv Res 2008;43(5 Pt1):1722-36.

30 Klein N, Bartlett J, Rowhani-Rahbar A, Fireman B, Baxter R. Waning protection after fifth dose of acellular pertussis vaccine in children. N Engl J Med 2012;367:1012-9.

31 Olin P, Hallander HO, Gustafsson L, Reizenstein E, Storsaeter J. How to make sense of pertussis immunogenicity data. Clin Infect Dis 2001;33(Suppl 4):S288-91.

32 Witt MA, Katz PH, Witt DJ. Unexpectedly limited durability of immunity following acellula pertussis vaccination in preadolescents in a North American outbreak. Clin Infect Dis 54:1730-5.

33 Orenstein EW, De Serres G, Haber MJ, Shay DK, Bridges CB, Gargiullo P, et al. Methodologic issues regarding the use of three observational study designs to assess influenza vaccine effectiveness. Int J Epidemiol 2007;36:623-31.

\section{Accepted: 27 June 2013}

\section{Cite this as: BMJ 2013;347:f4249}

This is an Open Access article distributed in accordance with the Creative Commons Attribution Non Commercial (CC BY-NC 3.0) license, which permits others to distribute, remix, adapt, build upon this work non-commercially, and license their derivative works on different terms, provided the original work is properly cited and the use is non-commercial. See: http://creativecommons.org/licenses/by-nc/3.0/. 


\section{Tables}

Table 1| Characteristics of people tested for pertussis, Kaiser Permanente Northern California, January 2006 to December 2011. Values are numbers (percentages) unless stated otherwise

\begin{tabular}{|c|c|c|c|}
\hline Characteristics & PCR positive cases (total=668) & PCR negative controls (total=10 098) & P value* \\
\hline \multicolumn{4}{|l|}{ Childhood pertussis vaccination history: } \\
\hline Pre-vaccine era & $61(9.1)$ & $1888(18.7)$ & \multirow[t]{5}{*}{$<0.001$} \\
\hline All whole cell vaccines & $129(19.3)$ & $4303(42.6)$ & \\
\hline First 3 doses whole cell vaccines & $191(28.6)$ & $2397(23.7)$ & \\
\hline $\begin{array}{l}\text { First } 3 \text { doses unknown or mix of whole cell and } \\
\text { acellular vaccines }\end{array}$ & $106(15.9)$ & $685(6.8)$ & \\
\hline All acellular vaccines & $181(27.1)$ & $825(8.2)$ & \\
\hline \multicolumn{4}{|l|}{ Year of PCR test: } \\
\hline 2006 & $85(12.7)$ & $1521(15.1)$ & \multirow[t]{6}{*}{$<0.001$} \\
\hline 2007 & $17(2.5)$ & $834(8.3)$ & \\
\hline 2008 & $22(3.3)$ & $718(7.1)$ & \\
\hline 2009 & $32(4.8)$ & $662(6.6)$ & \\
\hline 2010 & $393(58.8)$ & $4481(44.4)$ & \\
\hline 2011 & $119(17.8)$ & $1882(18.6)$ & \\
\hline Mean, SD (range) age (years) & $24.2,18.8(11-89)$ & $36.9,21.5(11-101)$ & $<0.001$ \\
\hline \multicolumn{4}{|l|}{ Age distribution (years): } \\
\hline $11-19$ & $466(69.8)$ & $3734(37.0)$ & \multirow[t]{7}{*}{$<0.001$} \\
\hline $20-29$ & $22(3.3)$ & $562(5.6)$ & \\
\hline $30-39$ & $30(4.5)$ & $1177(11.7)$ & \\
\hline $40-49$ & $53(7.9)$ & $1415(14.0)$ & \\
\hline $50-59$ & $37(5.5)$ & $1381(13.7)$ & \\
\hline $60-69$ & $41(6.1)$ & $1079(10.7)$ & \\
\hline$\geq 70$ & $19(2.8)$ & $750(7.4)$ & \\
\hline Male sex & $337(50.4)$ & 3669 (36.3) & $<0.001$ \\
\hline \multicolumn{4}{|l|}{ Race: } \\
\hline American Indian or Alaska Native & $4(0.6)$ & $72(0.7)$ & \multirow[t]{7}{*}{$<0.001$} \\
\hline Asian or Pacific Islander & $68(10.2)$ & $1281(12.7)$ & \\
\hline Black & $27(4.0)$ & $656(6.5)$ & \\
\hline Hispanic (regardless of race) & $128(19.2)$ & $1594(15.8)$ & \\
\hline Multiracial & $0(0.0)$ & $3(0.0)$ & \\
\hline White & $354(53.0)$ & $5579(55.2)$ & \\
\hline Imputed & $87(13.0)$ & $913(9.0)$ & \\
\hline
\end{tabular}

$\mathrm{PCR}=$ polymerase chain reaction.

${ }^{*} \mathrm{P}$ values are based on comparisons between PCR positive cases and PCR negative controls. $\mathrm{P}$ values were derived from $\mathrm{t}$ test for continuous age variable and from $x^{2}$ test for rest of variables. 
Table 2| Effectiveness of Tdap (reduced acellular pertussis) vaccination in preventing polymerase chain reaction (PCR) confirmed pertussis in selected subgroups defined by childhood pertussis vaccination history

\begin{tabular}{lcccc}
$\begin{array}{l}\text { Childhood pertussis vaccination history and } \\
\text { comparison group for PCR positive cases }\end{array}$ & No of cases & No of controls & $\begin{array}{c}\text { Vaccine effectiveness (\%) (95\% } \\
\text { Cl) }\end{array}$ & P value \\
\begin{tabular}{l} 
Pre-vaccine era: \\
\hline PCR negative controls*
\end{tabular} & 61 & 1887 & 24.1 (-58.7 to 63.7$)$ & 0.464 \\
\hline KPNC controls & 61 & 2725 & 26.7 (-65.4 to 67.5$)$ & 0.455 \\
\hline All whole cell vaccines: & 129 & & & $<$ \\
\hline PCR negative controls* & 129 & 4300 & 68.3 (45.8 to 81.5$)$ & $<0.001$ \\
\hline KPNC controls & & 4970 & 63.6 (37.3 to 78.9$)$ & $<0.001$ \\
\hline All acellular vaccines: & 181 & & & \\
\hline PCR negative controls* & 181 & 822 & 57.6 (34.1 to 72.7$)$ & $<0.001$ \\
\hline KPNC controls & 4776 & 74.4 (60.4 to 83.4$)$ & $<0.001$ \\
\hline
\end{tabular}

KPNC=Kaiser Permanente Northern California.

*Each of these models deleted a small number of observations for PCR negative controls because of missing covariate data (one observation in pre-vaccine model, three observations in all whole cell model, and three observations in all acellular model. 


\section{Figures}

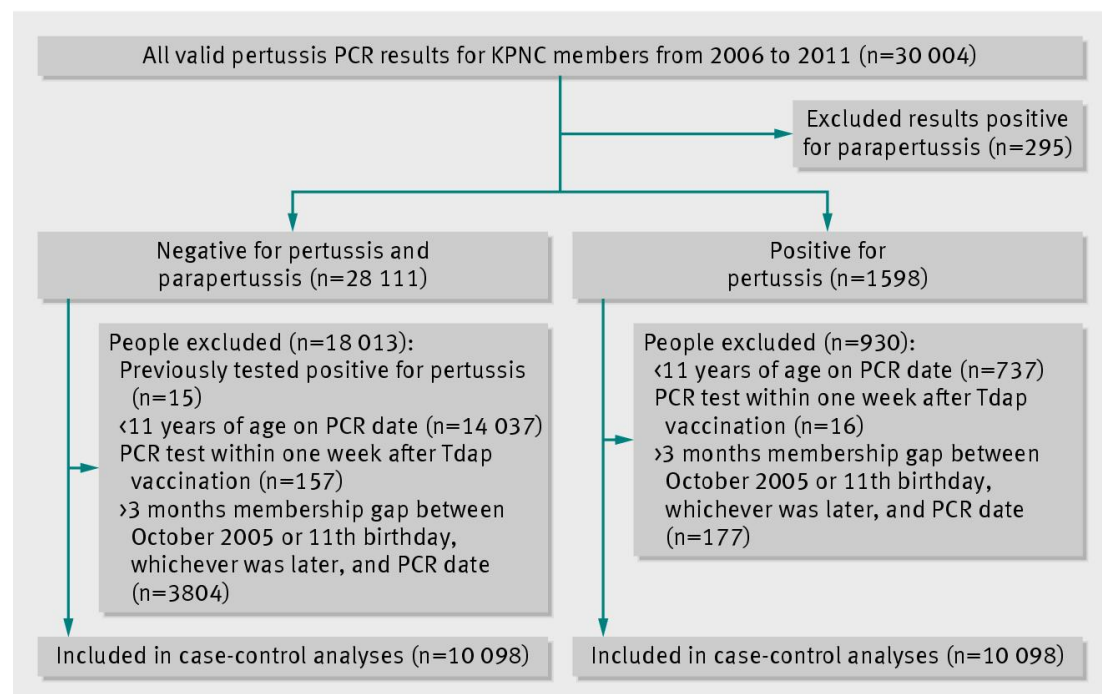

Fig 1 Inclusion and exclusion of cases and polymerase chain reaction (PCR) negative controls. January 2006 to December 2011, Kaiser Permanente Northern California (KPNC)

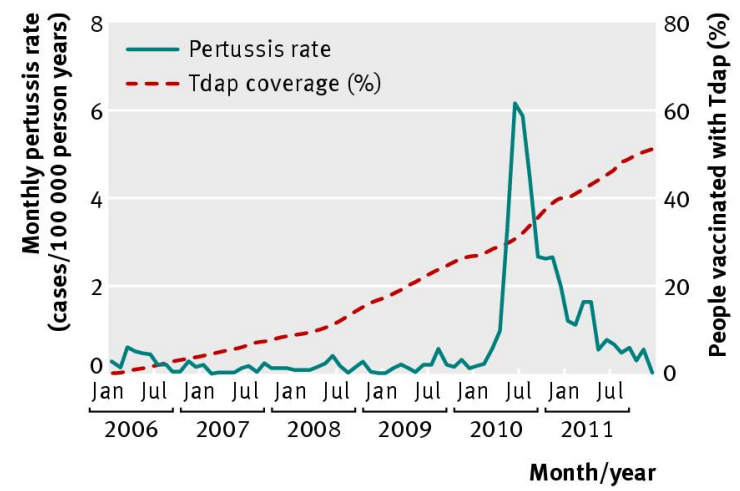

Fig 2 Pertussis incidence (all ages) and Tdap (reduced acellular pertussis) vaccination coverage (ages 11 and older), by month, January 2006 to December 2011, Kaiser Permanente Northern California 


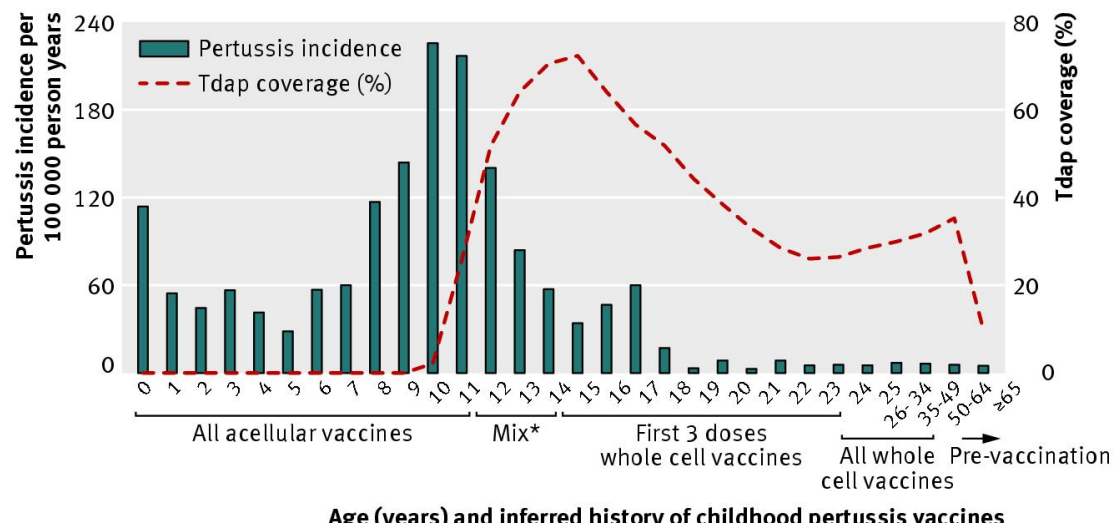

Fig 3 Pertussis incidence and Tdap (reduced acellular pertussis) coverage by age, January 2010 through June 2011, Kaiser Permanente Northern California. Incidence of pertussis for each age was calculated as: all cases of polymerase chain reaction (PCR) confirmed pertussis divided by all person years at risk and then multiplied by 100000 . Tdap vaccination coverage was calculated as of 14 August 2010, the median diagnosis date of cases during the 18 month period. Inferred history of childhood pertussis vaccines indicates the vaccine type that health plan members likely received in their childhood series based on their age on 14 August 2010. *Adolescents (12-14 years) with different pertussis vaccination histories (all acellular vaccines, first 3 doses a mix of acellular and whole cell vaccines, and first 3 doses whole cell vaccines) 